\title{
Terapia ocupacional neonatal, una propuesta para la acción
}

\author{
Mira O., Andrea ${ }^{[1]}$; Bastías L., Rodolfo ${ }^{[2]}$
}

\section{Resumen}

El trabajo en los Servicios de Neonatología requiere una adecuada comprensión de la compleja y bella relación que se da entre el Recién Nacido de Pretérmino, la familia y el equipo de salud.

Siendo esta un área donde nos encontraremos con una población muy susceptible y frágil, debemos propiciar que este niño sea capaz de responder adecuadamente a las demandas que este nuevo ambiente le presenta y así pueda madurar, crecer e ir adquiriendo los hitos del desarrollo de la mejor manera posible.

Para ello es importante implementar los Programas de Asistencia Individualizados y reconocer los aportes que la Terapia Ocupacional puede realizar en esta área.

Palabras Claves: Neonatología, Recién Nacido de Pretérmino, familia, equipo de salud, medio ambiente, Terapia Ocupacional.

\begin{abstract}
- Abstract
The work in the Neonatal Services requires an understanding of the complex and beautiful relationship between the newborn, the family and health staff.

Being this an area where we will find a very fragile and susceptible population, we must ensure the child's ability to respond appropriately to the demands imposed by this new environment, and in this way mature, grow and acquire the developmental milestones in the best possible way.

For this it is important to carry out Individualized Assistance Programs and to recognize the contributions of the Occupational Therapy in this area.
\end{abstract}

Key Words: Neonatology, Preterm Newborn, family, health staff, environment, Occupational Therapy.

\footnotetext{
${ }^{[1]}$ Terapeuta Ocupacional, Licenciada en Ciencias de la Ocupación Humana. Universidad de Chile. Diplomado en Neuropsicología, Universidad Católica, Formación en Integración Sensorial. Cargo Terapeuta Ocupacional Programa Integración Escolar Municipalidad Lo Barnechea. Centro Audiológico y de Trastorno de la Comunicación, "auditen". ${ }^{[2]}$ Kinesiólogo, Licenciado. Universidad de Chile. Bachiller en Ciencias Naturales y Exactas, Universidad de Chile. Kinesiólogo Servicio Neonatología, HSO. Diplomado "Formación en la Matriz Biológica de la Existencia Humana: Biología del Conocer y Biología del Amar". Instituto Matríztico, Ph. D. Humberto Maturana, Chile. Terapeuta Vojta, Internationale Vojta Gesellschaft e.V., Alemania, Formación en Integración Sensorial.
}

Contacto $>>{ }^{[1]}$ Mira O., Andrea: E-mail: andreamira@yahoo.com;

${ }^{[2]}$ Bastías L., Rodolfo: E-mail: rodolfobastias@hotmail.com 


\section{Introducción}

Es importante considerar que los Recién Nacidos de Pretérmino (RNPT) son una población cuya sobrevida ha ido en aumento, debido a los avances médicos y tecnológicos. Sin embargo, son un grupo susceptible de adquirir alteraciones en su desarrollo debido a su condición de prematuro.

Dada estas características por parte del RNPT, principalmente en su potencialidad de secuelas ulteriores, no sólo en el aspecto motor, también en lo cognitivo (aprendizaje escolar, conducta), es muy importante comenzar un plan de acción dentro de las Unidades de Neonatología, que para la gran mayoría de los niños será el punto de partida en la intervención en promoción de su desarrollo, debido a que por su condición estos después del alta continuaran sus intervenciones en los programas de seguimiento del prematuro respectivos $\underline{(18}, \underline{22}, \underline{23})$.

Profesionales tales como Terapeutas Ocupacionales, Kinesiólogos, y Psicólogos, entre otros, han logrado ingresar a estas unidades, abriendo un espacio, pasando de ser simples "invitados" a formar parte del equipo, ganándose la aceptación y confianza por medio de sus intervenciones y los resultados de éstas.

En los últimos años, a nivel mundial ha habido una evolución en cuanto a los parámetros para trabajar en esta área. Partiendo de un Modelo Rehabilitador, donde los RNPT eran derivados a Terapia Ocupacional ya sea por factores de riesgos específicos (bajo peso al nacer, consumo de drogas de los padres, etc.), diagnóstico de ciertas patologías (como asfixia severa y malformaciones congénitas), y/o por ciertos indicadores de desempeño (tono muscular anormal, problemas de alimentación, etc.). Los objetivos de intervención estaban dirigidos a problemas específicos, tales como limitaciones en los rangos de movimiento, irritabilidad, tono muscular alto o bajo 0 retrasos del desarrollo psicomotor.

Actualmente los parámetros para la intervención se han expandido más allá del Modelo Rehabilitador, a un enfoque que incluye conceptos de protección y prevención, con una mirada integral del niño, y de su relación activa con el medio ambiente físico y social ${ }^{(\underline{1}}$, 24).

Nuestro objetivo es dar a conocer las susceptibilidades del RNPT hospitalizado que pueden afectar su desarrollo y su relación con el medio, y mostrar como el Terapeuta Ocupacional puede desplegar sus herramientas profesionales y trabajar en equipo, a modo de favorecer y potenciar las habilidades de este niño para que pueda responder de manera adaptativa a las demandas del medio.

\section{Susceptibilidades del RNPT hospitalizado}

El desarrollo del neonato es producto de características individuales y de la experiencia en un contexto de maduración neurológica, salud y estabilidad fisiológica. Por ende, podemos afirmar que el desarrollo está determinado tanto por las particularidades genéticas como ambientales $(\underline{11})$. 


\section{Ambiente}

En el mundo intrauterino, el niño se encuentra en un ambiente tibio, oscuro, acogedor, con estímulos sonoros no invasivos, sin efecto directo de la gravedad, promoviendo un patrón flexor y entregándole los estímulos vestibulares, propioceptivos y tactiles que permiten una adecuada maduración y organización del Sistema Nervioso Central (SNC). Este ambiente satisface sus necesidades básicas, favoreciendo el comienzo del aprendizaje sensoriomotor y asentando las bases del apego. ${ }^{(1-4)}$

Los componentes del ambiente extrauterino de las Unidades de Neonatología son claramente diferentes a los que hay en útero. En este ambiente aparecen demandas como el respirar, regular la temperatura, moverse contra los efectos de la gravedad, ajustarse a la luz brillante y a los sonidos, lidiar con procedimientos invasivos y dolorosos, y con la frecuente deprivación de sueño.

Además de los anteriores, otros estímulos identificados y que dificultan la adaptación del RNPT, son el predominio del tacto médico versus el tacto social, posturas planas y horizontales, estimulación vestibular restringida o inadecuada, ruidos fuertes, mecánicos y constantes, luces encendidas durante todo el día y variaciones de la temperatura ambiental, etc. ${ }^{(1,5)}$

El SNC inmaduro del RNPT es competente para la vida intra uterina pero no está lo suficientemente desarrollada para ajustarse ni organizarse frente a los nuevos estímulos y demandas presentes, por lo cual la ocupación de este niño va estar enmarcada en el organizar su conducta, encontrando su estabilidad fisiológica y así poder relacionarse con el medio lo más favorablemente.

\section{Desarrollo sensoriomotor}

El desarrollo de los sistemas sensoriales comienza durante el primer trimestre de gestación, entran en funcionamiento en el segundo y en el tercero se produce una integración intensa de éstos. Paralelamente va desarrollándose el sistema motor, que junto a los componentes de la vida intrauterina (flexión, contención, línea media y comodidad, entre otros) facilitan un óptimo desarrollo, para permitir al neonato la más adecuada adaptación al medio. $(\underline{1}, \underline{11})$

Sin embargo, el desarrollo en RNPT se comporta de manera diferente a la de uno sin riesgo vital, dado que el proceso normal se ve interrumpido y su organismo debe producir cambios necesarios para la sobrevivencia, lo cual interfiere en la aparición esperada de los hitos de desarrollo. Por ejemplo, se sabe que en estos niños el nacimiento gatilla el desarrollo temprano de ciertas áreas, tales como respirar y digerir alimentos (leche), que algunas conductas serán inhibidas como el llevarse el pulgar a la boca y otras se verán alteradas como por ejemplo los patrones de movimiento.

El desarrollo sensoriomotor se relaciona directamente con los signos de dolor y estrés, por ello, diversos procedimientos médicos o de enfermería, en conjunto a un inadecuado manejo del dolor no farmacológico pueden llevar a aprendizajes inadecuados del prematuro. Esto se puede explicar porque, aún cuando la transmisión del dolor es funcional en el RNPT, los sistemas fisiológicos de protección son inmaduros, por lo cual el umbral de percepción del dolor es bajo, intenso y difuso, lo que hace que este niño muchas veces considere aquellos procedimientos como una mala experiencia, a pesar de que son vitales para su sobrevivencia. ${ }^{(\underline{2}, \underline{5})}$ 
Una inadecuada interacción del RNPT con el ambiente puede producir ciertos efectos a corto plazo (susceptibilidad a bradicardias, apneas, hipoxias, entre otras) y a largo plazo (por ejemplo, alteraciones de la percepción del dolor, trastornos del sueño, desarrollo motor inadecuado). Existen otras señales de alerta como las alteraciones del tipo succión - deglución y/o respiratorio, irritabilidad, pasividad, repertorio motor pobre, trastornos posturales, signos neurológicos suaves, no cumplimiento de hitos de desarrollo, deformaciones craneales, etc. (con o sin patología de base). Todos estos se deben tener presente en el momento de la evaluación, tratamiento o manejo. $(2, \underline{\underline{4}}, \underline{8}, \underline{1} \underline{\mathbf{9}})$

\section{Integración sensorial}

Las experiencias sensoriales son la puerta de entrada que nos permiten conocer e interactuar con el medio que nos rodea. Estas constituyen una poderosa fuerza capaz de modificar el estado general del organismo y tienen un gran impacto en el SNC. Este proceso de organización e interpretación de la información sensorial, que permite la adaptación del individuo a su entorno, es lo que se denomina Integración Sensorial. ${ }^{(11)}$

Las experiencias sensoriales a las que se está sometido desde la gestación tendrán un fuerte impacto en el desarrollo y en el como procesará e integrará futuros estímulos sensoriales. Es por esto, que es fundamental entregarles oportunidades a los RNPT de tener experiencias placenteras y confortables en relación a los diversos sistemas sensoriales. $^{(21)}$

Los sistemas táctil, propioceptivo y vestibular dominan las interacciones con el medio en etapas tempranas de la vida, siendo el momento más vulnerable durante el período de crecimiento cerebral rápido y de diferenciación neuronal entre las 28 y 40 semanas de gestación.

El feto dentro del útero está expuesto continuamente a estímulos tactiles, dado que los movimientos de la madre producen un masaje suave de presión profunda. Esta experiencia sensorial aplicada en RNPT suele tener efectos tranquilizadores. Por otro lado, el feto dentro del útero se encuentra suspendido en líquido y está expuesto a oscilaciones provocadas por él y por los movimientos de su madre. Los niños en las Unidades de Neonatología tienen pocas oportunidades de recibir experiencias sensoriales vestibulares que sean suaves y rítmicas, más bien, los movimientos que experimentan son impredecibles y repentinos, por lo que este sistema vestibular frágil es fácilmente sobrepasado, produciendo la aparición de efectos autonómicos. La información propioceptiva que recibe el feto es entregada por los movimientos que este realiza y por la contención que le da el medio. Sin embargo, esto cambia luego del parto, donde los inputs propioceptivos que recibe son mínimos. (211)

La Integración Sensorial es un acercamiento que reconoce y analiza la relación entre las demandas del medio ambiente y las respuestas fisiológicas del niño. A través de esta teoría podemos observar e interpretar sus conductas y capacidades.

El foco no está en la búsqueda de la adquisición de hitos del desarrollo, si no más bien, en el proceso a través del cual el niño va adquiriendo nuevas destrezas y como las utiliza para interactuar dinámicamente con el medio ambiente ${ }^{(25)}$.

Es importante destacar que los inputs sensoriales recibidos durante los primeros cuidados son uno de lo factores principales en el desarrollo del apego, dado que la 
relación afectiva entre padres e hijo está influenciada por la habilidad del niño para procesar y responder a los estímulos sensoriales.

Para lograr una adecuada Integración Sensorial no basta con tener un medio ambiente enriquecido (físico y social) sino que además debemos otorgar oportunidades al niño de autorregularse, modular sus respuestas, organizarse y producir Respuestas Adaptativas frente a las demandas del ambiente ${ }^{(25)}$.

\section{Respuesta adaptativa}

Tanto la deprivación como los excesos de estimulación van en detrimento del desarrollo, y en la mayoría de los casos, el SNC no logra ajustarse ni organizarse en este medio. En los RNPT una estimulación sensorial excesiva puede dañar a este cerebro que aun está en crecimiento ( $E j$.: hipoxias a repetición relacionadas con el estrés) y crear conductas desadaptativas, las que contribuirían a un desarrollo posterior pobre. ${ }^{(4)}$ Esto muestra la importancia de entregarle al neonato niveles de estimulación que pueda tolerar e integrar. $\left.{ }^{(\underline{3}, 11}\right)$

Es fundamental que el niño pueda recibir, procesar e integrar la información sensorial entrante y de este modo responder de manera adecuada a las demandas del ambiente. Esto es lo que podemos llamar Respuesta Adaptativa, considerándolas como fuerzas poderosas que dirigen el desarrollo hacia delante y que facilitan a su vez que el cerebro se organice y pueda producir respuestas cada vez más complejas. ${ }^{(11)}$. Las Respuestas Adaptativas favorecerán el desarrollo sensoriomotor del niño de pretérmino, dado que éstas son un ingrediente esencial para producir cambios positivos a nivel cerebral.

Las diversas intervenciones que realizan sobre los RNPT deben procurar que el niño logre y mantenga una adecuada estabilidad fisiológica, determinado por factores como un ritmo cardiaco estable, respiración suave y regular, color de piel saludable y una adecuada tolerancia alimentaria, entre otros. Este es el primer paso para que el niño pueda ser capaz de responder adaptativamente a las demandas del medio.

A medida que el niño madura va aumentando su capacidad para responder de manera organizada frente a los eventos que se le presentan, mostrándonos sus capacidades para autorregularse. Los RNPT, frente a los diversos estímulos van presentando conductas de evitación o búsqueda, estas últimas nos muestran sus esfuerzos para regularse. Son signos fundamentales para guiar y organizar nuestras intervenciones.

Dentro de las conductas de evitación tenemos el hipo, retorcerse, tendencia a la extensión de extremidades, llanto, movimientos desorganizados, entre otros. Éstas nos muestran que el niño requiere ayuda o un tiempo de tranquilidad. Por otro lado, las conductas de búsqueda, nos permiten determinar cuan competente es el niño para calmarse, explorar e interactuar socialmente; algunas de estas son el llevar la mano a la boca, el succionar, realizar movimientos suaves y coordinados, entre otros. $\left.{ }^{(6, \underline{6}, 21}\right)$ 


\section{El quehacer}

Tomando en consideración las susceptibilidades del RNPT aquí expuestas, desarrollaremos a continuación el quehacer, visto desde la mirada de un Programa de Asistencia Individualizada, en la identificación de las intervenciones del Terapeuta Ocupacional y en el trabajo en equipo con el Kinesiólogo.

\section{Programa de asistencia individualizada del RNPT}

De acuerdo a los antecedentes ya planteados, es sabido que variadas noxas que afectan al RNPT pueden ser evitables y otras controladas con la aplicación de un Programa de Asistencia Individualizada, basado en un programa certificado internacionalmente conocido como NIDCAP (Newborn Individualized Developmental Care and Assessment Programme), que tiene como objetivo final la facilitación de la adaptación extrauterina del neonato y proteger su estabilidad fisiológica. $\underline{(17, \underline{21})}$

Éste ha demostrado una serie de ventajas, como la disminución de los requerimientos de oxígeno adicional, menores días de apoyo ventilatorio, menor incidencia de apneas, mejor ganancia de peso, menos días de uso de sonda nasogástrica, mejor estado de organización, disminución en los costos y tiempo de hospitalización, mejorías en el comportamiento, desarrollo social y en el score de Bayley, entre otras ${ }^{(\underline{18})}$.

Creemos que es necesario la presencia de Terapeutas Ocupacionales y/o Kinesiólogos como coordinadores del programa, por lo que las Unidades de Neonatología deberían de disponer de sus servicios para llevar a cabo de manera integra su ejecución.

El objetivo es implementar medidas preventivas para las alteraciones que pueden aparecer producto de la discontinuidad del desarrollo fetal y facilitar el desarrollo del prematuro aspirando a que logre su máximo potencial.

Para ello debemos instruir a todo el equipo de salud en el reconocimiento de los signos de estabilidad fisiológica del RNPT y fomentar prácticas que lo favorezcan, reconocer los signos de estrés, tomando medidas para su disminución y promover la autorregulación. $\stackrel{(6,15)}{15}$

Con respecto al ambiente será fundamental incorporar en las Unidades de Neonatología los estándares internacionales de luminosidad y ruido. Utilizar luces cicladas y graduadas (10-600 lux), respetar ciclo día-noche utilizando cobertores, crear ambientes tranquilos en donde el ruido no exceda de un promedio de 45-65 decibeles y asegurar una adecuada mantención del ambiente térmico neutral.

Las atenciones de los diferentes profesionales deben estar coordinadas para respetar los periodos de descanso del niño y así evitar los excesos de manipulaciones y otros estímulos que puedan ser estresantes.

Asegurar en todo momento un adecuado posicionamiento, en donde el RNPT se mantenga alineado, contenido y favoreciendo la flexión. Evaluando la necesidad de utilizar elementos como nidos, soportes o el envolver con una manta al niño para facilitar la acción. 
Otro ítem importante de destacar es con respecto a los padres, en donde hay que otorgar las condiciones que promuevan el vínculo y apego, con adecuadas medidas

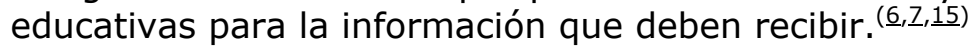

\section{Terapia ocupacional neonatal}

La orientación del quehacer de los Terapeutas Ocupacionales está relacionada con el favorecer un mejor Desempeño Ocupacional en el RNPT. Éste lo podemos observar en como el niño está lidiando con las demandas que implica el sobrevivir, crecer y desarrollarse. Por otro lado, debemos analizar y tratar de optimizar la relación recíproca entre el niño y el ambiente. El desempeño del neonato va a ser el resultado de la interacción entre habilidades intrínsecas (componentes de desempeño) y factores extrínsecos (variables ambientales).

Con respecto a la práctica esta debe ser respaldada con una adecuada formación en el área, identificando las condiciones médicas relevantes, procedimientos y equipos. Además de conocer las vulnerabilidades y las características específicas del desarrollo de los niños de pretérmino, el sistema familia y la ecología del lugar. ${ }^{(1,14)}$

Para facilitar una mayor comprensión del quehacer, es recomendado familiarizarse con las Teorías de Organización Neuroconductual, como por ejemplo, la Teoría Sinactiva de Heidelise Als, que provee las bases teóricas para comprender las conductas de los neonatos y los esfuerzos que éstos hacen para manejar el estrés del ambiente extrauterino. Otra teoría que nos puede orientar en las intervenciones es la de inturning, coming-out and reciprocity, desarrollada por Gorski, Davidson, y Brazelton, que describe los estados de los neonatos desde una respuesta autonómica hacia los estímulos, hasta que logran una interacción activa y recíproca con el ambiente. $(\underline{1}, \underline{6}, \underline{8})$

El trabajo de Heidelise Als, describe el desarrollo y diferenciación de 5 subsistemas: el Autonómico, el Motor, el de Estado y, el de Atención y Autorregulación. Señalando que la organización conductual se refleja en signos observables del funcionamiento de estos 5 subsistemas y el grado en que el niño puede autorregularse. Podemos analizar, entonces, el Desempeño Ocupacional del RNPT a través de estos Subsistemas y así con nuestras intervenciones trabajaremos para que logren madurar y desarrollarse lo más adecuadamente, pudiendo interactuar y responder de manera adaptativa a las

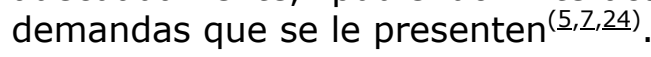

El combinar los principios de la teoría de Integración Sensorial con los de la Teoría Sinactiva nos permite comprender de mejor manera el desempeño del niño y así crear intervenciones más significativas para el niño y su familia ${ }^{(25)}$.

Los niños y la familia son vistos como una unidad integral, donde los padres toman un rol activo en el cuidado de sus hijos en las Unidades de Neonatología ${ }^{(24)}$.

\section{Evaluación}

Una evaluación integral y profunda es fundamental para lograr una apropiada intervención. Por ello y por los antecedentes ya expuestos, con los RNPT tener mayor cuidado y precaución en este proceso. Antes de evaluar al niño, debemos obtener 
información acerca de la Familia, la historia médica (información en relación al embarazo, los cuidados o problemas presentados, el parto y las complicaciones peri y postnatales) y la condición clínica actual del niño (procedimientos médicos recientes, complicaciones médicas no resueltas y nivel de homeostasis fisiológica) ${ }^{(1)}$.

Se debe observar las conductas espontáneas, sin la necesidad de estructurar el ambiente ni realizar maniobras especiales. Esto nos permite tener una mirada amplia acerca del niño y su interacción con el medio físico y social.

Además de las observaciones, se cuentan con pruebas de evaluación, donde hay una estructuración del ambiente y se realizan procedimientos previamente determinados. Estos nos permiten evaluar el desempeño del niño, identificar comportamientos normales o anormales y registrar los cambios. Algunas de las pautas que se utilizan son Naturalistic Observations of Newborn Behavioral (NONB), Assessment of Preterm Infant Behavioral (APIB), Neonatal Behavioral Assessment Scale (NBAS) y Observation on Espontaneous Movement (Prechtl) ${ }^{(21)}$.

Ya sea utilizando observaciones o pruebas de evaluación, se recomienda incluir los siguientes componentes dentro de este proceso: Ambiente (luz, sonido, nivel de actividad de la sala etc.), Organización Neuroconductual (estado de alerta, maduración neuroconductual, respuesta a estímulo), Desarrollo Neuromotor (desarrollo de reflejos, tono muscular, postura), Alimentación (desarrollo oro-motor) y Familia (vínculo familia-hijo, capacidad de los padres para participar activamente dentro de los cuidados neonatales). ${ }^{(1)}$

Se sugiere que la evaluación se programe en relación a los ciclos de sueño del niño, horarios de alimentación, rutinas de cuidado (cambio de pañales, higiene) y al estado clínico en que se encuentre.

\section{Intervención}

La intervención debe ser diseñada de manera individual para cada RNPT, logrando así adaptarse a sus necesidades, las que irán cambiando según su estado de salud, maduración y crecimiento, considerando en todo momento la estabilidad fisiológica ${ }^{(25)}$.

Los objetivos de las intervenciones deben ser considerados en la aplicación del Programa de Asistencia Individualizada, tanto en el fomento de la prevención y tratamiento. Se recalca la necesidad de trabajo en equipo, considerando las sugerencias de todos los profesionales que intervengan con el RNPT e incluir a la familia como entes activos dentro de los cuidados básicos del niño.

Dentro de las áreas a intervenir pueden señalarse:

\section{Modificaciones Ambientales}

Uno de los principales objetivos de la intervención es el evitar el estrés y el promover estados de estabilidad y calma. Esto lo podemos lograr a través sugerencias descritas en el Programa de Asistencia Individualizada (con respecto a luz, ruido, manipulaciones, etc). 


\section{Integración Sensorial}

Es muy importante entregar al RNPT experiencias sensoriales que sean placenteras y confortables y que faciliten el procesamiento adecuado de estímulos con el fin de promover la aparición de Respuestas Adaptativas.

Los estímulos tactiles, vestibulares y propioceptivos en esta etapa temprana de vida tienen un rol fundamental y son la base de un buen desarrollo sensoriomotor. Para ello, de acuerdo a las características de los RNPT, los estímulos deben ser dados de acuerdo a los umbrales de tolerancia del niño, por ejemplo, entregar solo un estímulo sensorial a la vez al inicio de la intervención, y luego ir incluyendo otros, de manera progresiva, a medida que vayan apareciendo Respuestas Adaptativas. Si se estimula al niño con demasiada información sensorial, sus SNC se verá sobrepasado y el niño no será capaz integrarla adecuadamente $\stackrel{(24)}{ }$.

Por ejemplo, podemos otorgar experiencias sensoriales de tacto profundo y estímulos propioceptivos pasivos (contención), las cuales han demostrado tener mejores resultados que las técnicas de masajes. Otra forma de entregar estímulos propioceptivos es a través de la facilitación de movimientos organizados, prehensión y succión, entre otras. También es importante otorgar inputs vestibulares lineales al sostenerlo en brazos y mecerlo, y realizar cambios de posiciones controladas (por ejemplo de decúbito supino a lateral). $\underline{(21, \underline{24})}$

Dentro de las Respuestas Adaptativas que esperamos observar es el lograr conductas motoras organizadas, tener suaves transiciones entre los estados de alerta, lograr un nivel de atención adecuado para interactuar socialmente y fortalecer su capacidad de autorregularse. $(\underline{1}, \underline{8}, \underline{21})$

\section{Neuromotora}

Es primordial ofrecer un adecuado posicionamiento que cumpla con los características intrauterinas (flexión, contención, línea media y confort) favoreciendo la organización del niño y evitando la aparición de deformidades anatómicas. ${ }^{(1,19,21)}$ Idealmente esto debe trabajarse en conjunto con el Kinesiólogo.

\section{Alimentación}

Velar por el cumplimiento de técnicas de alimentación (estimulación de succión nonutritiva, técnica de posicionamiento de alimentación, asesoramiento a madre durante la lactancia, etc.) que promuevan constantemente la estabilidad y autorregulación del niño durante esta actividad. ${ }^{(1,21)}$.

\section{Intervención con las familias}

En relación al trabajo con la familia, el rol fundamental de los Terapeutas Ocupacionales es facilitar una paternidad efectiva dentro del servicio y prepararlos para el alta. Promoviendo en todo momento la relación con su hijo(a), enseñándoles a reconocer los signos de estrés y estabilidad, dándoles apoyo durante el período de hospitalización, y guiándolos en el proceso de cuidados básicos (higiene, cambio de pañales, alimentación, etc.) ${ }^{(1)}$. 
Todo esto se realiza a través de la relación directa padres/ terapeuta, como un proceso informativo bidireccional, donde se debe estimularlos a participar activamente: compartiendo observaciones, tomando decisiones en conjunto, entre otros ${ }^{(\underline{1})}$.

La posibilidad de los padres de entregar los cuidados a sus hijos va a estar determinado por las planificaciones de atención de cada niño, su condición clínica y por las normas de cada establecimiento de $\operatorname{salud}^{(21)}$.

El hecho de tener a su hijo hospitalizado va a alterar sus ocupaciones y roles normales que relacionados con su vida familiar, trabajo, entre otros. Es importante considerar este aspecto, para incluirlo dentro de las posibles asistencias que debemos entregar a la familia.

\section{Trabajo en equipo con el kinesiólogo}

Tanto el Kinesiólogo como el Terapeuta Ocupacional deben permitirse el trabajo en conjunto, para así que cada intervención que realicen contengan los objetivos de cada especialidad, y así sumar los esfuerzos que promueven la adaptación del RNPT y su óptimo desarrollo sensoriomotor.

El alineamiento postural y el desarrollo del sistema músculo esquelético se consolida en cada postura experimentada por el RNPT. Un inadecuado posicionamiento puede conducir a asimetrías y deformidades de este sistema, además de afectar la organización neuroconductual y la función motora de estos niños ${ }^{(19)}$.

Por lo tanto, el Kinesiólogo debe proveer al RNPT un alineamiento estructural que le facilite el desarrollo funcional de la postura y el movimiento, así como el prevenir alteraciones sensoriomotoras, en especial en aquello niños con mayores factores de riesgo que son más susceptibles a un aprendizaje motor desorganizado (Por ejemplo, los que son sometidos a ventilación mecánica convencional).

Sus acciones abarcaran áreas de prevención, educación y tratamiento, tanto así en las intervenciones directas con el niño como en las sugerencias en el actuar de otros profesionales.

Para ello posee formación en las áreas de intervención motora y sensorial que le permitirán favorecer respuestas motoras al RNPT acordes a su edad gestacional corregida, y en el caso que correspondan, realizar tratamiento a los impedimentos que pueda presentar. Otro nivel de acción es el respiratorio, donde se buscan ciertos objetivos específicos como el optimizar su biomecánica respiratoria, prevenir o reexpandir atelectasias, permeabilizar vía aérea, etc. En muchas oportunidades estas características se dan en forma simultánea, teniendo que priorizar algunas acciones. $\stackrel{(20)}{ }$ 


\section{Reflexiones}

\section{Trabajo en equipo}

Una visión globalizada nos permitirá optimizar la intervención neonatal de todos los profesionales y de las familias que participen en el Servicio de Neonatología. Esta relación provoca el nacimiento de la aplicación de un Programa de Asistencia Individualizada Neonatal atingente a cada lugar de trabajo, que permite facilitar el desarrollo y adaptación del RNPT durante su estadía hospitalaria. Esto incluye: enriquecer las prácticas en pro del desarrollo integral del niño de pretérmino, moderar el impacto sensorial ambiental y la inclusión de la participación activa de los padres. Si bien, en otros lugares del mundo se realizan este tipo de programas (como NIDCAP) no debemos pretender grandes proyectos en donde los recursos son insuficientes. El comienzo del programa se vería favorecido realizando una ampliación de la mirada hacia el tema, sobre el rol de la familia y las ventajas de éste.

El Equipo de Salud en conjunto con el Terapeuta Ocupacional, determinarán de mejor manera como abordar al RNPT, compartiendo sus miradas en una visión global del neonato y sus necesidades, donde éste es considerado un ser activo dentro de la relación y donde su bienestar no está determinada por elementos aislados, sino más bien por una compleja interacción entre su medio interno y el externo (físico y social).

\section{Familia}

Este enfoque, nos orienta necesariamente a lo que es la interacción más importante a nivel neonatal: la Familia. El comprender esta mirada, nos llevará a un viaje más placentero que solo el hecho de evocarnos a eventos aislados.

Las familias se ven repentinamente enfrentadas a un mundo totalmente nuevo, con un lenguaje desconocido y lejano. Donde su hijo está recibiendo los cuidados necesarios para poder sobrevivir, sin embargo, es en este punto donde ellos se ven atrapados en una red de sensaciones de miedo y ansiedad que les dificulta ver y reconocer sus capacidades para hacerse cargo de este hijo y asumir su rol de padres.

Es por esto que necesitarán de una guía y apoyo para pasar por las diversas etapas (hospitalización, alta, etc.). En este proceso se crea un dialogo entre los padres, el niño y el equipo de salud, desarrollando una mejor capacidad de comunicación y comprensión entre estos. Lo que se refleja en la habilidad de los padres de observar y comprender las conductas de su hijo y a la vez, la recepción activa por parte de este último, de los cuidados que le entregan.

Con todo lo anterior iremos preparando tanto a los padres como al neonato para una adecuada alta. Donde será crucial acompañarlos en este proceso de transición del Hospital a sus hogares, ya que pasaran a ser los responsables del bienestar de su hijo.

Reforzamos el hecho de ver el quehacer como una experiencia para el niño, familia y equipo de salud, observando que es un camino no exento de dificultades, por ende, cada intervención promoverá, de alguna u otra manera, un adecuado desarrollo en un ambiente optimizado, colaborando para un mejor porvenir del niño. 
Mientras damos cuenta de la importancia de las adaptaciones que ocurren en el RNPT y también en aquellos que interactúan con él, la adaptación aparece como un juego de sobrevivencia hermoso de distinguir y quizás el ejercicio con mayores frutos para nuestros ámbitos de atención en salud.

\section{Referencias}

(1) Hunter J.G. The Neonatal Intensive Care Unit. En Case-Smith J., Allen A., Pratt P., Occupational Therapy (1996). $3^{\circ}$ edición Editorial Mosby; 583-631.

(2) Porter FL, Wolf CM, Miller J.P. The effect of handling and inmobilization on the response to acute pain in newborn infants. Pediatrics $1998 ; 102(6): 1383-9$

(3) Lester B., Tronick E. Stimulation and the preterm infant: the limits of plasticity. Clinical in Perinatology, $1990 ; 17(1)$

(4) Grunau R. Early Pain in preterm infants. A model of long term effects. Clinical in Perinatology, 2002; 29: 373-394

(5) Perlman J. Neurobehavioral deficits in premature graduates of intensive carepotential medical and neonatal environmental risk factors. Pediatrics, 2001; 108(6): 1339-48.

(6) Als, H. (1986). A synactive model of neonatal behavior organization: framework for the assessment of neurobehavioral development in the premature infant and for support of infants and parents in the neonatal environment. Physical and Occupation Therapy in Pediatrics, 6,3-55.

(7) Als H. Et al.. "Early experience alters brain function and structure".Pediatrics; 113(4).April 2004,pp.846-857.

(8) Gorski, P.A., Davidson, M.F., \& Brazelton, T.B. (1979). Stages of behavioral organization in the high-risk neonate: theoretical clinical considerations. Seminars in Perinatology, 3,61-72

(9) Amiel-Tison C. Neurología Perinatal (2001). Editorial MASSON S.A.

(10) Msall et al. Functional outcomes in self-care, mobility, conmunication, and learning in extremely low-birth weight infants. Clinical in Perinatology, 2000; 27(2): $381-401$. 
(11) Parham D., Mailloux Z. Sensory Integration. En Case-Smith J., Allen A., Pratt P., Occupational Therapy (1996). $3^{\circ}$ edición Editorial Mosby; 307-356

(12) Guerrero A.. "Desarrollo psicológico de los niños nacidos prematuros o de muy bajo peso". Boletin Sociedad Psiquiatria y Neurología Infantil y Adolescencia. Año10; 2:38-43

(13) White-Traut, RC., Nelson, MN., Silvestri, JM., Patel, MK., Kilgallon, D. Patterns of physiological and behavioral response of intermediate care preterm infant to intervention. Pediatrics Nursery, 1993;19:625-629

(14) American Occupational Therapy Association (1993) Knowledge and skills for occupational therapy practice in the neonatal intensive care unit. American Journal of Occupational Therapy, 47,1100-1105.

(15) Feldman R., Eidelman A. Intervention Programs for premature infants. Clinical in Perinatology, 1998; 25: 613-626.

(16) Buehle D.M. et al. Effectiveness of individualized developmental care for the low risk preterm infants: behavioral and electrophysiologial evidence.

Pediatrics, 1995; 96(5): 923-932.

(17) Resnick, MB., Amstrong, S., Carter, RL. Developmental intervention program for high-risk premature infants: Effects on development and parent-infant interactions. Journal Developmental Behavioral Pediatrics, 1988; 9:73-78

(18) Revisión Sistemática Cochrane. Disponible en www.nichd.nih.gov/cochraneneonatal/cochrane.cfm

-Symington A, Pinelli J. Developmental care for promoting developmental and preventing morbidity in preterm infant (Consultado Marzo de 2006)

-Conde-Agudelo A., Diaz-Rosello J.L., Belizan J.M. Kangoroo mother care to reduce morbidity and mortality in low birthweight infants. (Consultado Marzo de 2006)

-Vickers A., Ohlsson A., Lacy JB., Horsley A. Massage for promoting growth and development of preterm and7or low birth-weight infants. (Consultado Marzo de 2006)

-Pinelli J., Symington A. Non-nutritive sucking for promoting physiologic stability and nutrition in preterm infants. (Consultado Marzo de 2006)

(19) Sweeney, J., Gutierrez, T. Musculoskeletal Implications of Preterm Infant Positioning in the NICU. Journal of Perinatal and Neonatal Nursing. 58- 70, 
June 2002.

(20) Figueroa P. Estándares y Normativas para la prestación de servicios en Kinesiología Neonatal. Disponible en www.prematuros.cl (Consultado en Mayo 2006)

(21) Warren, I. Guidelines for Infant Development in the Newborn Nursery. Handbook developed for the Winnicott Baby Unit al St. Mary's NHS Trust in London. $3^{a}$ Edition 2001

(22) "Programa de seguimiento de Prematuros, Chile". Disponible en www.prematuros.cl (Consultado en Septiembre 2006)

(23) Avilés, C., Madariaga, P., Fuentes, P., et al. Intervención Temprana en prematuros, una experiencia de trabajo. Revista Chilena de Terapia Ocupacional Número 4, Noviembre 2004, pp. 11-18

(24) Hunter J.G. The Neonatal Intensive Care Unit. En Case-Smith J., Allen A., Pratt P., Occupational Therapy (2001). $4^{\circ}$ Edition Mosby; 636-707.

(25) Schaaf, R., Anzalone, M. Sensory Integration with High-Risk Infants and Young Children. En Smith, S., Imperatore, E., Schaaf, R. Understanding the Nature of Sensory Integration with Diverse Populations (2001). Editorial Therapy Skill Builders; 275-312. 